\title{
EL LARGO CAMINO HACIA LA GESTIÓN INTEGRAL DE CRISIS EN LA UNIÓN EUROPEA
}

\author{
Guillem Colom ${ }^{1}$
}

Universidad Pablo de Olavide

\begin{abstract}
Resumen:
Las operaciones de gestión de crisis realizadas desde el fin de la Guerra Fría han puesto de manifiesto la necesidad de armonizar la cooperación multinacional para incrementar la coherencia de la operación. Éste es el principio fundamental del Enfoque Integral, definido como la concertación de las estrategias y las acciones de los actores participantes en la gestión de crisis a todos los niveles y planos de la misma. Desde 2008, la Unión Europea está definiendo su propio modelo para mejorar su unidad de acción en materia de gestión de crisis, ampliar su cooperación práctica con el resto de los actores implicados en la resolución de la misma y apoyar el desarrollo de capacidades civiles y militares. El trabajo analiza sus antecedentes y estado actual.
\end{abstract}

Palabras clave: Gestión de crisis, enfoque integral, cooperación civil-militar, coordinación civil-militar,

Política Común de Seguridad y Defensa.

Title in English: "The Long Way towards Comprehensive Crisis Management in the European Union"

\begin{abstract}
:
The crisis management operations carried out since the end of the Cold War have highlighted the need to harmonize multinational cooperation as a means to enhance the coherence and effectiveness of the mission. This is the fundamental principle of the Comprehensive Approach, defined as the synchronization of the strategies and actions of the actors involved in the crisis management at all levels and phases. The European Union has been defining since 2008 its own Comprehensive Approach in order to improve its comprehensiveness in foreign and security policies; to expand its practical cooperation with other relevant actors in crisis management operations and to support the development of civilian and military capabilities. The article analyses the background and current developments of the European Comprehensive Approach.
\end{abstract}

Keywords: Crisis Management, Comprehensive Approach, Civil-Military Cooperation, Civil-Military Coordination, Common Security \& Defence Policy.

Copyright @ UNISCI, 2014.

Las opiniones expresadas en estos artículos son propias de sus autores, y no reflejan necesariamente la opinión de UNISCI. The views expressed in these articles are those of the authors, and do not necessarily reflect the views of UNISCI

\footnotetext{
1 Profesor del área de ciencia política y de la administración de la Universidad Pablo de Olavide y del departamento de relaciones internacionales de la Universidad Pontificia de Comillas.

E-mail: gcolpie@upo.es.

http://dx.doi.org/10.5209/rev_UNIS.2013.n34.4483ם
} 


\section{Introducción}

Hoy en día es difícil hallar una sola operación de asistencia humanitaria, gestión de crisis, estabilización posconflicto o construcción nacional que no se desarrolle en un marco multinacional, en la que no participen numerosas agencias u organizaciones o no requiera emplear abundantes herramientas de tipo político, diplomático, económico, humanitario, policial o militar.

Y es que la experiencia acumulada tras años de participación en operaciones de este tipo no sólo ha revelado la dificultad que entraña la gestión y resolución de unas crisis cada vez más complejas tanto en su dimensión interna como en su vertiente exterior; sino que también ha puesto de manifiesto la necesidad de concertar las estrategias, capacidades y acciones de los distintos actores desde el primer momento y al más alto nivel, compartiendo - en la medida de lo posible - información, situación final deseada, objetivos estratégicos, planeamiento operativo y gestión de recursos. Esta coordinación debe abarcar desde el nivel estratégico al táctico y realizarse en todas las fases de la operación, desde su inicial concepción y posterior planteamiento hasta su ejecución sobre el terreno y final evaluación.

La solución propuesta para realizar eficazmente este tipo de labores en un ambiente multinacional e interagencias recibe el nombre de Enfoque Integral (Comprehensive Approach) y se fundamenta en la armonización de los objetivos, las estrategias, las capacidades y las actividades de los distintos participantes a todos los niveles, planos o fases de la operación.

La Unión Europea no ha sido ajena a estos desarrollos en la esfera de la seguridad, y desde el año 2008 también está definiendo su propio modelo de Enfoque Integral para resolver sus carencias en materia de seguridad, ampliar la más restringida Coordinación CivilMilitar, explotar la nueva arquitectura institucional perfilada por el Tratado de Lisboa y unificar definitivamente la vertiente civil y militar de su acción exterior y dimensión de seguridad y defensa.

Teniendo estos elementos en cuenta, el presente artículo examinará los antecedentes, evolución y estado actual del Enfoque Integral europeo, la más ambiciosa iniciativa - tras una Cooperación Civil-Militar encaminada a mejorar la colaboración ad-hoc a nivel táctico y una Coordinación Civil-Militar para mejorar la coherencia interna de la Unión Europea en toda la gama de misiones Petersberg - propuesta por Bruselas para mejorar su unidad de acción en materia de prevención y gestión de crisis, ampliar su cooperación práctica con el resto de los actores implicados en la resolución de la misma y apoyar el desarrollo de capacidades civiles y militares al servicio de su dimensión exterior, de seguridad y de defensa.

\section{El concepto de Enfoque Integral}

Calificado como el nuevo paradigma para la prevención de conflictos y la gestión de crisis internacionales, el concepto de Enfoque Integral ${ }^{2}$ fue concebido a mediados de la pasada década para explicar la multidimensionalidad de los riesgos a la seguridad internacional y dar

\footnotetext{
${ }^{2}$ No obstante, antes de que se impusiera el concepto de Comprehensive Approach, se barajaron varias ideas con distintos matices como Enfoque Global (Global Approach), Planeamiento y Acción Concertados (Concerted Planning \& Action), Acción Gubernamental (Whole-of-Government Approach), Enfoque 3D (3D Approach) o Seguridad en Red (Networked Security).
} 
respuesta a las dificultades observadas durante la conducción de operaciones de asistencia humanitaria, gestión de crisis, mantenimiento de la paz, estabilización posconflicto o construcción nacional que se han venido realizando desde $1989^{3}$.

En términos generales, la necesidad de desarrollar nuevas herramientas nacionales y multinacionales para la gestión de crisis se puso de manifiesto tras el fin de la Guerra Fría, cuando muchos conflictos que habían permanecido latentes tras la política de bloques estallaron con toda su violencia y obligaron a la Comunidad Internacional a intervenir en su resolución. Desde entonces, el grueso de las operaciones de este tipo se han planteado en un entorno normativo y político difuso; se han realizado en un marco multinacional complejo; han contado con la participación de numerosos actores (fuerzas armadas, agencias gubernamentales, organizaciones internacionales, empresas privadas u ONGs) con cometidos, agendas, objetivos y recursos muy dispares; y han requerido también la concertación de una amplia gama de acciones e instrumentos de naturaleza política, diplomática, económica, informativa, humanitaria, policial o militar ${ }^{4}$.

Estas experiencias han expuesto las carencias nacionales e internacionales en materia de gestión de crisis, estabilización posconflicto o construcción nacional. Por un lado, pronto se observó que los ejércitos occidentales - equipados, adiestrados, adoctrinados y organizados para combatir en una hipotética guerra contra la Unión Soviética y el Pacto de Varsovia gozaban de una supremacía militar en el plano convencional, pero que en muchos casos carecían de las capacidades necesarias para conducir operaciones de baja y media intensidad o llevar a cabo labores de estabilización o prestar apoyo militar a las tareas de reconstrucción. Esta carencia comportó el desarrollo de nuevas capacidades militares y la reorientación de las existentes para realizar con éxito este conjunto de operaciones muy alejadas de las tradicionales misiones de los ejércitos.

Igualmente, la creciente presencia de actores civiles (agencias gubernamentales, organizaciones internacionales, ONGs, empresas privadas o medios de comunicación) en el teatro de operaciones comportó el desarrollo de nuevos protocolos de actuación y colaboración, la codificación de la Cooperación Civil-Militar - que también constituye el primer paso del Enfoque Integral europeo - y el desarrollo de nuevos métodos de planeamiento concertado o de empleo limitado de medios civiles bajo mando militar 5 .

Y por último lugar, las recientes experiencias en labores de estabilización y construcción nacional en escenarios hostiles como Afganistán e Irak no sólo han obligado a limitar las aspiraciones de la Comunidad Internacional en la creación de Estados y descartar al menos por el momento - nuevas aventuras de este tipo; revelado la inviabilidad política y estratégica de mantener largas campañas militares; expuesto el valor de la comunicación estratégica y la gestión de la información pública u obligado a revisar los pilares de la

\footnotetext{
${ }^{3}$ Friis, Karsten y Jarmyr, Pia (eds.) (2008): Comprehensive Approach. Challenges and opportunities in complex crisis management, Oslo, Norwegian Institute of International Affairs.

4 Kent, Randolph: "International humanitarian crises - two decades before and two decades beyond", International Affairs, vol. 80, $\mathrm{n}^{\circ} 5$ (octubre 2004), pp. 851-869 o Weiss, Thomas: "Learning from military civilian intervention on peace operations", International Peacekeeping, vol. 6, no 2 (verano 1999), pp. 112-128.

5 Entre estos desarrollos destacan la Acción Unificada (Unified Action) estadounidense, entendida ésta como el empleo de medios militares conjuntos pertenecientes a un Mando Combatiente en apoyo a operaciones multinacionales e interagencias (Joint Chiefs of Staff [JCS] (2008): JP-02 Unified Action Armed Forces, Washington DC, U.S. Government Printing Office); o las Operaciones Basadas en Efectos (Effects Based Operations) que, reemplazadas por el Enfoque Integral, pretendían integrar, desde el primer momento y al más alto nivel, el esfuerzo militar y no-militar. Para más información, véase: Colom, Guillem: "La evolución de la concepción operativa basada en efectos”, Política y Estrategia, no 117 (junio-diciembre 2011), pp. 61-77.
} 
contrainsurgencia y elaborar un nuevo cuerpo de pensamiento para acometer las labores de estabilización y apoyo militar a la reconstrucción; sino que también han mostrado la extrema necesidad de integrar los esfuerzos - civiles y militares, públicos y privados o nacionales e internacionales - en un proceso de análisis, planeamiento, ejecución y evaluación lo más integrado y homogéneo posible ${ }^{6}$.

Y para solventar estos graves problemas que dificultan la prevención y gestión de crisis, el Enfoque Integral pretende concertar las estrategias, capacidades y actividades de todos los participantes en la resolución del conflicto, desde el primer momento y al más alto nivel, y compartiendo - en la medida de lo posible - inteligencia, situación final deseada, objetivos estratégicos, planeamiento operativo y asignación de recursos. Esta coordinación debe comprender desde el nivel político-estratégico al táctico, y deberá realizarse en todas las fases y planos de la operación: desde su concepción inicial y posterior planeamiento operativo hasta su ejecución sobre el terreno y final evaluación ${ }^{7}$.

Esta integración vertical y horizontal de las actividades debe permitir que los participantes en la gestión de la crisis dispongan de un procedimiento de concertación abierto (que les dote de un conocimiento compartido de la situación y de sus posibles cursos de evolución); inclusivo (que garantice la asistencia y facilite las contribuciones humanas y materiales de todos los actores interesados en participar); transparente (para que los distintos participantes conozcan el funcionamiento del proceso de toma de decisiones, planeamiento y evaluación); ágil (porque no exista posibilidad de bloqueo y las discrepancias internas puedan ser resueltas por la misma organización) y realista (porque la situación final deseada y los objetivos a conseguir en la operación dependan de las prioridades y de los medios compartidos durante la fase de concertación) ${ }^{8}$.

El éxito del Enfoque Integral depende de la voluntad, determinación y solidaridad de los participantes, y su implementación requiere transformar la cultura organizativa y el funcionamiento interno de las instituciones dedicadas a la gestión de crisis. No obstante, es probable que una vez el empleo de este modelo se haya generalizado entre la Comunidad Internacional, éste dificulte la reproducción de los errores cometidos por nuestros países en los Balcanes, Afganistán, Irak o Libia, tales como la definición de objetivos políticos demasiado ambiciosos, inalcanzables, ambiguos o irreales; la improvisación en los planteamientos estratégicos; la rivalidad institucional de los participantes; la ignorancia de los códigos culturales de los escenarios de actuación; la falta de comunicación entre los actores militares y los civiles, nacionales e internacionales o públicos y privados sobre el terreno; la generación de estructuras para la gestión de crisis redundantes e ineficientes; la dispersión de los recursos humanos y materiales, la exclusión de ciertos actores clave en el proceso de toma de decisiones o la tendencia de los actores militares por interferir en el espacio humanitario 9 .

\footnotetext{
${ }^{6}$ De Carvalho, Benjamin y Aune, Ingrid (2010): Assessing Complex Peace Operations, Some Considerations of Methodology and Procedure, Oslo, Norwegian Institute for International Affairs o Rotman, Philipp (2010): Built on shaky ground: the Comprehensive Approach in Practice, Research Paper nº 63, Roma, NATO Defence College.

7 Bensahel, Nora; Oliker, Olga y Peterson, Heather (2009): Improving Capacity for Stabilization and Reconstruction Operations, Santa Monica, RAND Corporation.

${ }^{8}$ Rinatokski, Kristina. y Autti, Mikko (eds.) (2008): Comprehensive Approach: Trends, Challenges and Possibilities for Cooperation in Crisis Prevention and Management, Helsinki, Crisis Management Initiative o VV.AA. (2008): Multinational Experiment 5 (MNE5): Report of Synthesized Findings, Suffolk, United States Joint Forces Command.

${ }^{9}$ El espacio humanitario - un entorno en el que las actividades humanitarias y de desarrollo se realizan libremente, con imparcialidad y neutralidad - ya se ha visto amenazado en numerosas ocasiones debido a la violencia e inseguridad imperante en la zona de operaciones y la patente ingerencia militar en labores
} 
Sin embargo, a pesar de las bondades teóricas y enorme potencial práctico de esta iniciativa, a fecha de hoy no se ha consensuado ninguna definición de Enfoque Integral que pueda ser aceptada entre la Comunidad Internacional ${ }^{10}$; todavía no existe ningún acuerdo sobre su configuración a nivel multinacional ${ }^{11}$ y tampoco se ha propuesto un modelo único de concertación interagencias a nivel nacional ${ }^{12}$. En consecuencia, las concepciones existentes de Enfoque Integral incluyen formalmente estos principios pero su principal objetivo no es integrar las capacidades de todos los participantes en la gestión de una crisis internacional, sino resolver los problemas internos de la propia organización y facilitar, en la medida de lo posible, la coordinación con otros actores presentes en el área de crisis ${ }^{13}$.

Como puede observarse, los modelos presentados permiten constatar que cada país dispone de su propia fórmula específica para coordinar a sus departamentos y agencias, así como sus propios procedimientos de actuación para incrementar su coherencia interna: desde la más simple y orientada a la cooperación práctica entre los ministerios de defensa, asuntos exteriores y cooperación al desarrollo, hasta la plena integración de todas las administraciones del estado y la participación de actores privados y la sociedad civil.

\section{El Enfoque Integral Europeo}

Desde un punto de vista formal, la Unión Europea está especialmente capacitada para utilizar un Enfoque Integral en su acción exterior. Su concepción integral y extensiva de la seguridad, su vocación multilateralista, su predilección por el poder blando, la amplia gama de instrumentos a su disposición y una Política Común de Seguridad y Defensa (PCSD) fundamentada en la concertación de las capacidades militares y civiles, deberían hacer del Comprehensive Approach algo natural y característico de la Unión Europea y el rasgo

humanitarias. En este sentido, no parece extraño que desde el ámbito humanitario se tema que el Enfoque Integral pueda utilizarse para condicionar, politizar o militarizar la asistencia humanitaria, amenazando así la independencia, neutralidad e imparcialidad de la ayuda prestada No obstante, empleado con racionalidad y coherencia, el Enfoque Integral proporciona a todos los participantes una parcela de responsabilidad y una esfera de actuación específicos, lo que se traduce en un espacio humanitario independiente y seguro para estos actores. En este sentido, véase: Cormish, Stephen y Glad, Marit (2008): Civil-Military Relations: No room for Humanitarism in Comprehensive Approaches, Oslo, Norwegian Atlantic Committee.

${ }^{10}$ Petersen, Fris y Binnendijk, Hans: "From Comprehensive Approach to Comprehensive Capability", NATO Review, no 3 (marzo 2008), en http://www.nato.int/docu/review/2008/03/ART7/EN/index.htm.

${ }^{11}$ En este sentido, mientras la Alianza Atlántica está desarrollando un Enfoque Integral que pretende mejorar su funcionamiento interno y la cooperación con otros actores relevantes en el planeamiento, conducción y evaluación de operaciones de gestión de crisis no-Artículo 5; las Naciones Unidas están implementando las Misiones Integradas para incrementar la coordinación interna de sus agencias en el análisis y planeamiento de operaciones de paz; y la Caja de Herramientas de Planeamiento para asistir a los actores sobre el terreno en el análisis, planeamiento, gestión y administración de la misión. Para más información, véase: De Conning, Cedric (2007): Coherence and Coordination in United Nations Peacebuilding and Integrated Missions, Oslo, Norwegian Institute for International Affairs; Department of Peacekeeping Operations [DPKO], (2012): Planning Toolkit, Nueva York: Organización de las Naciones Unidas o Colom, Guillem: "La evolución del Enfoque Integral aliado para la gestión de crisis”, Afers Internacionals, no 97 (abril 2012), pp. 287-304.

${ }^{12}$ En este sentido, las concepciones nacionales tienden a fundamentarse en los modelos 3D (diplomacia, defensa y desarrollo); 3D+C (diplomacia, defensa, desarrollo y comercio) o Enfoque Gubernamental (participación de todos los ministerios y agencias del Estado), siendo imposible hallar ningún ejemplo práctico de Enfoque Integral (coordinación interministerial y colaboración de la sociedad civil, ONGs o empresas privadas). Patrick, Stewart y Brown, Kaysie (2007): Greater than the Sum of its Parts? Assessing "Whole-of-Government Approaches" to Fragile States, Nueva York, International Peace Academy.

${ }^{13}$ Rotman, op. cit., pp. 7-9. 
distintivo tanto de su dimensión de seguridad y defensa ${ }^{14}$ como de su acción exterior ${ }^{15}$. No obstante, fue necesario esperar hasta 2008 para que Bruselas emprendiera el desarrollo formal de su propia concepción integral para resolver sus carencias manifiestas en materia de seguridad y defensa, explotar la arquitectura institucional perfilada por el Tratado de Lisboa y unificar de forma definitiva la dimensión civil y militar de su acción exterior.

A pesar de ello, los orígenes del Comprehensive Approach europeo pueden fijarse con la articulación de la Política Europea de Seguridad y Defensa (PESD) y sus pilares conceptuales en la Estrategia Europea de Seguridad del año $2003^{16}$. Por un lado, el lanzamiento de esta iniciativa comportó la creación de los organismos necesarios para su funcionamiento $^{17}$; la definición del catálogo de operaciones a realizar (las Misiones Petersberg $)^{18}$; la identificación de los objetivos de capacidades militares y civiles para asumir sus compromisos (los Headline Goals) ${ }^{19}$; el establecimiento de los compromisos para suplir carencias específicas (los acuerdos Berlín Plus con la Alianza Atlántica) y la conducción de veinticinco misiones, diez de las cuales de naturaleza civil y cuatro de tipo civil-militar.

${ }^{14}$ De hecho, Javier Solana, Alto Representante para la Política Exterior y de Seguridad Común, aseveró que:
"The Comprehensive Approach underpinning ESDP is its value added. The logic underpinning ESDP - its
distinctive civil-military approach to crisis management - was ahead of its time when conceived. That logic has
proved its validity and has been adopted by others." Solana, Javier (2009): "Remarks by the EU High
Representative for the Common Foreign and Security Policy", ESDP@ 10: What Lessons for the Future?, París,
Instituto de Estudios de Seguridad de la Unión Europea, París, p. 3; Esta misma idea también la defiende Karl
Von Wogau, presidente del subcomité de seguridad y defensa del Parlamento Europeo entre 2004 y 2009 :
Wogau, Karl V. (ed.) (2009): The Path to European Defence. New roads, New Horizons, Londres, John Harper
Publishing, Londres, p. IX.
15 De hecho, asumiendo las provisiones del Tratado de Lisboa en materia de acción exterior, son cada vez más
las voces que consideran el Enfoque Integral como el fundamento de la política exterior europea. Ejemplos de
ello pueden encontrarse en los trabajos de Woollard, Catherine (2013); The EU and the Comprehensive
Approach, Bruselas, European Peace Building Liaison Office y Stephan Lehne: "Promoting a Comprehensive
Approach to EU foreign policy", Carnegie Europe, 21 febrero 2013, en http://carnegieeurope.eu/2013/02/21/promoting-comprehensive-approach-to-eu-foreign-policy/fio o las declaraciones de las autoridades europeas.

${ }^{16}$ Gross, Eva (2008): EU and the Comprehensive Approach. DISS Report 2008-13, Copenhagen, Danish Institute for International Studies.

${ }^{17}$ La puesta en marcha de la PESD comportó la creación de un Comité Político y de Seguridad encargado tanto de la dirección estratégica y el control político de las operaciones como de garantizar la armonización entre la vertiente civil y militar de la gestión de crisis; un Comité para los Aspectos Civiles de la Gestión de Crisis que informaría y asesoraría al anterior en todo lo referente a la dimensión civil de la gestión de crisis; un Comité Militar con la misión de realizar las recomendaciones militares pertinentes al anterior y establecer las directrices que implementará el Estado Mayor, responsable del planeamiento y conducción de las operaciones.

${ }^{18}$ Definidas por la Unión Europea Occidental en 1992 y codificadas en el Artículo 17 del Tratado de Niza, las Misiones Petersberg contemplan operaciones humanitarias o de rescate; prevención de conflictos y mantenimiento de la paz; gestión de crisis que precisen el empleo de fuerzas de combate; labores de desarme; asistencia militar y estabilización posconflicto. A este conjunto de misiones se le sumarán años después coincidiendo con la presentación de la Estrategia Europea de Seguridad - las operaciones conjuntas de desarme, de apoyo a terceros países en materia antiterrorista o de reforma del sector de seguridad.

${ }^{19}$ A grandes rasgos, en el Consejo Europeo de Helsinki (1999) se aprobó el Helsinki Headline Goal, que determinaba las capacidades militares y civiles requeridas para el funcionamiento de la PESD. Mientras las primeras se fijaron en 60.000 efectivos, las segundas - especificadas durante el Consejo Europeo de Feira (2000) - se establecieron unos objetivos de 5.000 policías, 282 expertos en materia judicial; 160 efectivos en administración civil y 2.000 miembros de protección civil, todos ellos preparados para actuar en labores de prevención de conflictos y gestión de crisis. Aunque este desarrollo de capacidades está siendo más lento y difícil de lo que cabría esperar, se pretende que la Unión disponga de un catálogo de capacidades militares y civiles que le doten de una cierta capacidad de acción autónoma y le permitan garantizar la consecución de los objetivos fijados por la Estrategia Europea de Seguridad. 
Por otro lado, la Estrategia Europea de Seguridad de 2003 fue el primer documento en subrayar la necesidad de integrar los distintos instrumentos a disposición de la Unión Europea para enfrentarse con éxito a cualquier riesgo o amenaza que pudiera cernirse sobre el continente $^{20}$. Además, la estrategia proclamaba su determinación en favorecer un "multilateralismo eficaz" basado en la actuación de la Unión Europea junto con otros actores - en especial las Naciones Unidas, la Alianza Atlántica o la Organización para la Seguridad y Cooperación en Europa - para construir “...una sociedad internacional más fuerte, con instituciones internacionales que funcionen adecuadamente y un orden internacional basado en el Derecho." ${ }^{21}$. Dicho de otra forma, el "Documento Solana" ya reconocía una realidad que ha terminado por convertirse en uno de los pilares - al menos desde una perspectiva formal - de la acción exterior europea: que los riesgos y las amenazas a la paz y la seguridad internacionales requieren un esfuerzo coherente de la Unión Europea que integre el empleo de todos los instrumentos a su disposición (políticos, diplomáticos, militares, económicos, policiales, cooperación al desarrollo, etc.) y su colaboración con otros actores internacionales relevantes.

\subsection{De la cooperación civil-militar a la coordinación civil-militar}

Al igual que el Comprehensive Approach aliado, el Enfoque Integral europeo tiene sus antecedentes más remotos en las Guerras de los Balcanes. Estos conflictos pusieron de manifiesto tanto la enorme brecha militar que existía entre Estados Unidos y el resto de sus aliados y la conveniencia de dotar a la Unión Europea de una dimensión de seguridad y defensa; como la necesidad de mejorar la atención de las fuerzas militares hacia la población civil o sistematizar las labores y definir las responsabilidades entre los actores militares y civiles de la operación. Y mientras la primera carencia condujo al lanzamiento de la PESD vinculada al desarrollo de mecanismos civiles y militares de gestión de crisis $^{22}$; la segunda motivó la creación de la primera gran herramienta para reforzar la cooperación práctica entre los elementos civiles y militares en a escala táctica.

Originalmente concebida para aunar el planeamiento y la conducción de las labores militares y civiles en los niveles estratégico y operacional ${ }^{23}$, la Cooperación Civil-Militar (Civil-Military Cooperation o CIMIC) fue elaborada por el Estado Mayor de la Unión Europea y adoptada por el Consejo a comienzos de $2002^{24}$. No obstante, este concepto acabó regulando - tal y como había planteado un año antes la Directiva 411/1 del Comité Militar de la Alianza Atlántica (Military Policy on Civil Military Co-operation) ${ }^{25}$ e institucionalizó el

\footnotetext{
${ }^{20}$ Según reza el documento, “...ninguna de las nuevas amenazas es meramente militar, ni puede atajarse únicamente con medios militares. Cada una de ellas requiere una combinación de instrumentos." (Consilium: Una Europa Segura en un Mundo Mejor: Estrategia Europea de Seguridad, 12 diciembre 2003, p. 14. Esta misma idea también puede observarse, entre otros documentos, en el informe sobre la implementación de la estrategia (Consilium: Informe sobre la aplicación de la Estrategia Europea de Seguridad: Ofrecer seguridad en un mundo en evolución, S407/08, 11 diciembre 2008.

${ }^{21}$ Consilium: "Una Europa Segura...", op. cit., p. 9. En este sentido, téngase en cuenta que esta reivindicación del "multilateralismo eficaz" también se debía a la coyuntura del momento, marcada por la publicación de la controvertida Estrategia Nacional de Seguridad estadounidense de 2002, de naturaleza unilateralista y basada en la acción preventiva.

22 Una visión general puede hallarse en: Pérez de las Heras, Beatriz y Churruca, Cristina (2009): Las capacidades civiles y militares de la UE: estado de la cuestión y propuestas de cara a la Presidencia Española 2010, Documento de Trabajo no 41/2009, Madrid, Fundación Alternativas.

${ }^{23}$ Gebhard, Caarmen (2008): Civil-Military Coordination and Cooperation in the Context of the EU's Crisis Management: CMCO vs. CIMIC, Vienna, Institute for Peace Support and Conflict Management - National Defence Academy o Gross, op. cit., pp. 13-17.

${ }^{24}$ Consilium: CIMIC Concept for EU-led Crisis Management Operations, 7106/02, 18 marzo 2002.

${ }^{25}$ NATO Military Committee [MC]: MC 411/1 Military Policy on Civil-Military Cooperation, 18 enero 2002.
} 
documento doctrinal AJP-9 (NATO Civil-Military Coordination) ${ }^{26}$ un año después - la coordinación ad-hoc sobre el terreno de los elementos militares y los actores civiles externos a la Unión Europea (organizaciones internacionales, ONGs, población civil o autoridades locales) para incrementar la protección de las fuerzas militares en la zona de operaciones ${ }^{27}$.

La presentación de la Cooperación Civil-Militar europea comportó el establecimiento de unidades militares especializadas en CIMIC y su incorporación en las operaciones de gestión de crisis; y coincidió con el compromiso del Comité Político y de Seguridad de impulsar otras fórmulas de colaboración mucho más ambiciosas y susceptibles de ser empleadas en toda la gama de las operaciones Petersberg. Sin embargo, mientras las autoridades europeas estaban proyectando la Coordinación Civil-Militar (Civil-Military Coordination o CMCO); también presentaron un modelo tentativo de planeamiento integral para la gestión de crisis; introdujeron una célula de coordinación civil-militar para garantizar la coherencia de la planificación estratégica ${ }^{28}$; establecieron medidas de colaboración práctica entre los pilares y agencias europeas en los niveles político y estratégico durante todo el ciclo de la operación e implementaron fórmulas específicas para mejorar la coordinación civil-militar europea sobre el terreno ${ }^{29}$.

Por un lado, a mediados de 2003 se propusieron un conjunto de Procedimientos para la Gestión Coherente e Integral de las Crisis (Procedures for Coherent, Comprehensive Crisis Management $)^{30}$ con el objetivo de reforzar la colaboración entre el Consejo y la Comisión en la planificación y financiación de las operaciones, garantizar el liderazgo del Comité Político y de Seguridad en la coordinación de los aspectos civiles y militares de la crisis, y facilitar las relaciones entre los actores en la conducción y evaluación de las mismas. Dos años después, esta guía sería reemplazada por el Concepto para el Planeamiento Integral (Concept for Comprehensive Planning) de todas las operaciones PESD. Definida como una herramienta fundamental para la consecución de la Coordinación Civil-Militar ${ }^{31}$ y proyectada para apoyar su implementación práctica en línea con los procedimientos anteriores, cubrir el ciclo de planeamiento y sistematizar la cooperación práctica de todos los participantes en la misión, esta iniciativa proponía “...un enfoque sistemático que garantice la coordinación de las actividades intra e inter pilares de todos los actores relevantes de la Unión Europea en el planeamiento de las operaciones de gestión de crisis." 32 . En consecuencia el Planeamiento Integral pretendía que los actores y agencias europeas participaran junto con los Estados miembros en la puesta en común de sus estimaciones sobre la crisis e integraran sus estrategias, concertaran sus planes sectoriales, armonizaran sus actividades sobre el terreno y

\footnotetext{
${ }^{26}$ NATO Standardisation Agency [NSA] (2003): AJP-9 NATO Civil-Military Cooperation (CIMIC) Doctrine, Bruselas, OTAN.

${ }^{27}$ Un análisis más detallado de la cooperación civil-militar puede hallarse en Haugevik, Kristin y Carvalho, Benjamin (2007): Civil-Military Cooperation in Multinational and Interagency Operations, Security in Practice $\mathrm{n}^{\mathrm{o}} 2$, Oslo, Norwegian Institute for International Affairs.

${ }^{28}$ Integrada en el Estado Mayor de la Unión Europea, la controvertida Célula Civil-Militar (CivMil Cell) fue originalmente planteada en el año 2003 para paliar la limitada presencia de la dimensión civil en materia de alerta temprana, análisis de situación, provisión de opciones estratégicas o planificación civil-militar de las operaciones PESD (Kohl, Radek: "Civil-Military Coordination in EU Crisis Management" en Nowak, Agnieszka (ed.) (2006): Civilian Crisis Management: The EU Way, Chaillot Paper n 90, París, European Union Institute for Security Studies, pp. 123-136 o Pullinger, Stephen (ed.) (2006): Developing EU Civil Military coordination: the Role of the New Civilian Military Cell, Bruselas, ISIS Europe - CeMISS, pp. 6-8 y 11-18.

${ }^{29}$ Gebhard, op. cit., pp. 13-14 o Gross, op. cit., pp. 21-23.

${ }^{30}$ Consilium: Suggestions for Procedures for Coherent, Comprehensive EU Crisis Management, 11127/03, 3 julio 2003.

31 Drent, Magriet: “The EU's Comprehensive Approach to Security: a Culture of Coordination?", Studia Diplomatica, vol. LXIV, no 2 (verano 2011), pp. 3-18 o Gebhard, op. cit., pp. 15-17.

${ }^{32}$ Consilium: Draft EU Concept for Comprehensive Planning, 13983/05, 3 noviembre 2005, p. 2.
} 
evaluaran el impacto de las mismas con el fin de lograr la situación final deseada por la Unión Europea $^{33}$. No obstante, aunque estas guías podrían haber servido para incrementar la unidad de acción europea en materia de gestión de crisis y orientar el desarrollo de su Enfoque Integral, hasta la fecha su impacto real ha sido menor de lo deseado. Estas iniciativas nunca han sido formalmente aceptadas, sus provisiones no son de obligado cumplimiento y no han servido para regular la coordinación de las herramientas a disposición de la Comisión y el Consejo, tampoco han sentado las bases para desarrollar nuevos procedimientos y nunca se han empleado para planificar las misiones PESD debido al limitado interés que todavía muestran las estructuras europeas de gestión de crisis ${ }^{34}$.

Por otro lado, a finales del año 2003 se presentó el concepto de Coordinación CivilMilitar $^{35}$ para “...coordinar de manera efectiva las acciones de todos los actores relevantes de la Unión Europea implicados en la planificación e implementación de la respuesta europea a la crisis." ${ }^{36}$. Considerada por varios expertos como el arquetipo del Enfoque Integral europeo ${ }^{37}$ o la translación de esta idea al proceso de planeamiento operativo de las misiones PESD ${ }^{38}$; la Coordinación Civil-Militar pretendía incrementar la coordinación respetando siempre la independencia, objetivos e integridad de todos los participantes - entre los pilares y agencias europeas en el planeamiento, conducción y evaluación de las operaciones europeas de gestión de crisis. Igualmente, esta iniciativa pretendía sentar las bases de una nueva "cultura de coordinación" que, fundamentada en la armonización de los objetivos, la concertación de los medios y la cooperación práctica en todas las fases de la operación, transformara la cultura organizativa de las instituciones europeas ${ }^{39}$ y gobernara las relaciones entre los elementos civiles y militares de la Unión Europea en la resolución de conflictos $^{40}$.

Y es que en materia de gestión de crisis, la tradicional complejidad institucional de la Unión Europea y las ambiguas relaciones entre la estructura civil y militar ha obligado a Bruselas a lograr un difícil equilibrio entre los intereses políticos y estratégicos de los Estados miembros, las concepciones - a veces opuestas - del Consejo y la Comisión y las inercias

\footnotetext{
${ }^{33}$ Es interesante apuntar que esta misma idea fue planteada por Francia en el año 2003 para definir la coordinación interministerial durante todo el ciclo de respuesta a crisis (Bagayoko, Niagalé y Kovacs, Anne (2007): La gestion interministérielle des sorties de conflits, París, Sciences Sociales de la Défense).

${ }^{34}$ Para un mayor conocimiento de estas propuestas y sus limitaciones prácticas, véase: Hansen, Annika (2006): Against All Odds - The Evolution of Planning for ESDP Operations. Civilian Crisis Management from EUPM onwards, Berlín, Center for International Peace Operations, o Kohl, op. cit., pp. 126-128.

${ }^{35}$ Este concepto no debe confundirse con la Civil-Military Coordination (CMCoord) de las Naciones Unidas, una iniciativa cuyo fin es armonizar - en la medida de lo posible - las labores realizadas por las agencias de esta organización, las llevadas a cabo por otros actores humanitarios y las operaciones conducidas por las fuerzas militares internacionales.

${ }^{36}$ Consilium: Civil-Military Coordination (CMCO), 14457/03, 7 noviembre 2003, p. 1.

${ }^{37}$ A pesar de limitarse a la vertiente interna de la Unión Europea - algo que el Comprehensive Approach debe trascender si pretende establecer medidas de colaboración con otros actores externos - son varios los autores que asimilan la Coordinación Civil-Militar con el Enfoque Integral. En este sentido, véase: Gebhard, op. cit., p. 13; Knutsen, Bjørn (2008): The EU and the challenges of Civil-Military Coordination at the Strategic Level, Oslo, Norwegian Defence Research Establishment; Major, Claudia y Mölling, Christian: "More than Wishful Thinking? The EU, UN, NATO and the Comprehensive Approach to Military Crisis Management", Studia Diplomatica, vol. LXII, no 3 (otoño 2009), pp. 21-29; Norheim-Martinsen, Martin (2009): Matching Ambition with Institutional Innovation: The EU's Comprehensive Approach and Civil-Military Organisation, Oslo, Norwegian Defence Research Establishment; Hynek, Nik (2010): Consolidating the EU's Crisis Management Structures: Civil-Military Coordination and the Future of the EU OHQ, EXPO/B/AFET/FWC/2009-01, Bruselas, Parlamento Europeo o Pullinger, op. cit., pp. 12-19.

${ }^{38}$ Drent, op. cit., p. 8.

${ }^{39}$ Ibid., p. 4.

${ }^{40}$ Para conocer las diferencias entre la CIMIC europea y la CMCO, véase Kohl, op. cit., pp. 123-128.
} 
provocadas por la división de esta organización en tres grandes pilares hasta la entrada en vigor del Tratado de Lisboa. No obstante, los responsables de elaborar la Coordinación CivilMilitar evitaron abrir esta Caja de Pandora y centraron sus esfuerzos en establecer unas pautas mínimas e informales de coordinación entre los pilares de la Unión y reforzar la complementariedad entre las atribuciones del Consejo (que tiene a su disposición las herramientas precisas para planear y conducir las operaciones y los medios necesarios para sostener las labores de estabilización a largo plazo) y la Comisión (que goza de competencias presupuestarias en materia de política exterior y ayuda al desarrollo, se responsabiliza de las delegaciones sobre el terreno y puede prestar una asistencia de impacto pero limitada en tiempo y espacio) $)^{41}$.

Aunque desde el año 2003 se han realizado grandes avances en el desarrollo teórico e implementación práctica de la Coordinación Civil-Militar - caso de la presentación de un Concepto para la Gestión de Crisis que enmarca esta iniciativa ${ }^{42}$; un Concepto para el Planeamiento Integral que la sistematiza; un documento de debate que la desarrolla ${ }^{43}$; un conjunto de medidas que guían su aplicación práctica ${ }^{44}$ e incluso una célula Civil-Militar en el seno del Estado Mayor de la Unión Europea que debería velar por su consecución ${ }^{45}$ - es también cierto que esta iniciativa que busca mejorar la colaboración intra e inter pilares e incrementar la coordinación de los elementos civiles y militares a disposición de la Unión Europea en el marco de la PCSD continúa siendo hoy en día objeto de controversia ${ }^{46}$. Y es que la entrada en funcionamiento de la Coordinación Civil-Militar no ha servido para superar las inercias de la PESD e incrementar la coordinación interna de la Unión Europea en materia de gestión de crisis; ni ha reforzado las relaciones entre sus elementos civiles y militares durante el planeamiento y la conducción de las operaciones; ni tampoco ha sentado las bases para la generación de un catálogo de capacidades civiles-militares adecuado y utilizable en la prevención de conflictos y la gestión de crisis ${ }^{47}$. Igualmente, la "cultura de coordinación"

\footnotetext{
${ }^{41}$ En un plano formal, los Equipos de Coordinación de Respuesta a Crisis (Crisis Response Coordination Teams) - grupos creados ad-hoc para preparar el concepto de gestión de crisis y velar por la integración de los aspectos civiles y militares en todos los niveles y planos de la operación - garantizarían que el Consejo y el Secretariado de la Comisión abordan de manera integral la crisis.

${ }^{42}$ Consilium: EU Concept for Military Planning at the Political and Strategic Level, 10687/08, 16 junio 2008.

${ }^{43}$ El non-paper titulado Enhancing EU Civil Military Co-Ordination fue presentado por el Reino Unido, Austria y Finlandia - los países que formarían la troika comunitaria a partir del segundo semestre de 2005 - y pretendía impulsar el desarrollo de esta iniciativa en cinco grandes áreas: valoración previa de la crisis; planeamiento estratégico integrado; gestión de la operación; definición de métricas para evaluar su progreso y gestión de capacidades. Aunque no es accesible públicamente, una referencia al mismo puede hallarse en: Pullinger, op. cit., p. 17.

${ }^{44}$ Las medidas prácticas diseñadas para apoyar la implementación de esta iniciativa en toda la gama de las operaciones PESD comprendían el diseño de una estrategia integral para todos los actores europeos, la definición de un plan de actividades coherente, la provisión de apoyos cruzados entre los distintos organismos, la sincronización de las labores a realizar sobre el terreno o la elaboración de una política de comunicación e información pública coherente y coordinada entre las agencias europeas. Consilium: Civil-Military Coordination (CMCO) Framework for Possible Solutions for the Management of EU Crisis Management Operations, 8926/06, 2 mayo 2006.

${ }^{45}$ Aunque su creación se debe a otros condicionantes y su utilidad real ha sido sensiblemente menor de lo esperado, la Célula Civil-Militar podría haber facilitado significativamente la consecución práctica de la Coordinación Civil-Militar.

${ }^{46}$ Driver, Darrell: "The European Union and the Comprehensive Civil-Military Approach in Euro-Atlantic Security: Matching Reality to Rhetoric”, Strategic Studies Quarterly, vol. 4, no 3 (otoño 2010), pp. 136-155; Drent, Margriet y Zandee, Dick (2010): Breaking Pillars: Towards a civil-military security approach for the European Union, CSP, nº 13, La Haya, Clingendael; Kohl, op. cit., pp. 128-136 o Drent, op. cit., 8-13.

${ }^{47}$ Ibid., pp. 7-9; Driver, op. cit., pp. 141-143; Kohl, op. cit., pp. 129-131; Gebhard, op. cit., 18-23 o Menon, Anand y Sedelmeyer, Ulrich: "Instruments and Intentionality: Civilian Crisis Management and Enlargement Conditionality in EU Security Policy”, West European Politics, vol. 33, nº 1 (enero 2010), pp. 75-92.
} 
promovida por esta iniciativa ha tenido un impacto mucho menor de lo esperado: al basarse en el voluntarismo de las partes por establecer vínculos de colaboración ad hoc - algo motivado por las reticencias en aceptar la imposición de protocolos formales que requirieran la adopción de compromisos específicos entre los actores ${ }^{48}$ - esta coordinación de las actividades europeas en todas las fases y planos de la gestión de crisis ha permanecido limitada y el planeamiento integral entre los elementos civiles y militares no se ha logrado ${ }^{49}$.

Estas carencias volvieron a poner de manifiesto las dificultades que debe afrontar la Unión Europea a la hora de implementar mecanismos propios para la gestión de crisis; los obstáculos que impiden la integración práctica de las vertientes civil y militar de su dimensión exterior; o las limitaciones inherentes de su arquitectura institucional, que dificulta las relaciones entre las estructuras, dilata los ciclos de planeamiento, entorpece la financiación de las operaciones y compromete la coherencia y sostenibilidad de las mismas en el largo plazo. En consecuencia, Bruselas estimó necesario lanzar nuevas iniciativas para armonizar la acción inter e intra pilares, simplificar la cadena de mando de las operaciones civiles e incrementar la colaboración práctica entre los elementos civiles y militares a disposición de la PESD; y establecer también unos estándares mínimos comunes entre los veintisiete sobre cómo acometer la gestión de crisis.

Estas iniciativas confluyeron en la constitución de una Capacidad de Planeamiento y Conducción Civil (Civilian Planning and Conduct Capability o CPCC) ${ }^{50}$ encargada del planeamiento, despliegue, conducción, mando, control y evaluación de las operaciones civiles de gestión de crisis; el establecimiento de un Directorio para el Planeamiento y la Gestión de Crisis (Crisis Management and Planning Directorate o CMPD) ${ }^{51}$ con la misión de facilitar a las autoridades políticas europeas una visión integral de la crisis y responsabilizarse del planeamiento civil-militar en el nivel estratégico ${ }^{52}$; y la integración de ambos organismos junto con el Estado Mayor y el Centro de Situación - dentro del Servicio Europeo de Acción

\footnotetext{
48 de Vasconcelos, Álvaro; Grevi, Giovanni; Helly, Damien y Keohane, Daniel (eds.) (2009): European Security and Defence Policy: The First 10 Years (1999-2009), París, European Union Institute for Security Studies, Paris, pp. 19-68 o Schroeder, Ursula: "Governance of EU Crisis-Management", en Emerson, Michael y Gross, Eva (eds.) (2007): Evaluating the EU Crisis Missions in the Balkans, Bruselas, Centre for European Policy Studies, pp. 17-45.

${ }^{49}$ Ioannides, Isabelle: "EU Civilian Capabilities and Cooperation with the Military Sector", en Greco, Ettore; Pirozzi, Nicoletta y Silvestri, Stefano (eds.) (2010): EU Crisis Management: Institutions and Capabilities in the Making, Roma, Istituto Affari Internazionali, pp. 29-54.

${ }^{50}$ Dependiente del Directorado General para la Gestión de Crisis Civiles (DG E IX) y considerado como la contraparte civil de la cadena de mando militar, este organismo fue establecido en el año 2007 tras la aprobación de una hoja de ruta que establecía las funciones, los cometidos y las responsabilidades del Comandante para las Operaciones Civiles (CivOpCdr). Véase: Consilium: Guidelines for Command and Control Structure for EU Civilian Operations in Crisis Management, 10381/07, 17 junio 2007. Un análisis más detallado del mismo puede hallarse en: Gross, op. cit., pp. 23-25 o Drent y Zandee, op. cit., pp. 19-22.

${ }^{51}$ Creado a finales de 2008 mediante la fusión del Directorado General para la Gestión de Crisis Civiles (DG E IX) con el Directorado General para Asuntos de Defensa (DG E VIII), este órgano se sitúa bajo la autoridad de la DG E (Asuntos Exteriores y Político-Militares) del Secretariado del Consejo. Consejo Europeo: Declaration of the European Council on the Enhancement of the ESDP (Anexo 2), 12 diciembre 2008. Un análisis más detallado de sus funciones puede hallarse en: Gebhard, Carmen: "The Crisis Management Planning Directorate. Recalibrating ESDP Planning and Conduct Capacities", CFSP Forum, vol. 7, no 4 (abril 2009), pp. 8-14.

${ }^{52}$ Entre otros cometidos, este organismo es el responsable de elaborar el Concepto de Gestión de Crisis (Crisis Management Concept) que será elevado a las autoridades políticas europeas y sentará las bases del proceso de planeamiento operativo. Es interesante comentar que éste guarda enormes similitudes con la propuesta francesa de Planeamiento Multinacional Interagencia (Multi-National Interagency Strategic Planning) que se evaluó durante el Experimento Multinacional 5 realizado entre los años 2006 y 2008. Un análisis más detallado del mismo puede hallarse en VV.AA. (2009): Key Elements of a Comprehensive Approach: A Compendium of Solutions, Suffolk, United States Joint Forces Command, pp. IV.1-IV.23.
} 
Exterior que, bajo la autoridad del Alto Representante para Asuntos Exteriores y Política de Seguridad, coordinaría los instrumentos, unificaría las cadenas de mando y lideraría las actividades de la Unión Europea en materia de gestión de crisis y resolución de conflictos ${ }^{53}$.

\subsection{El Tratado de Lisboa v el Enfoque Integral}

$Y$ en este contexto marcado por los cambios institucionales motivados por la entrada en vigor del Tratado de Lisboa, “...que brinda nuevas oportunidades para explotar y coordinar el potencial de la PCSD junto con otros instrumentos y sectores de la acción exterior de la Unión Europea para lograr el Enfoque Integral" ${ }^{\text {"5 }}$ y el establecimiento del Servicio Europeo de Acción Exterior, “...que contribuirá de manera decisiva al desarrollo de un Enfoque Integral europeo a la gestión de crisis civiles y militares, prevención de conflictos y construcción de la paz" "55, fueron las autoridades militares las que emprendieron el desarrollo de un Comprehensive Approach que complementara y ampliara la más restringida Coordinación Civil-Militar, todavía considerada por algunos como el modelo de Enfoque Integral europeo ${ }^{56}$.

Basándose en las líneas trazadas por el Plan para el Desarrollo de Capacidades (Capability Development Plan) ${ }^{57}$ de 2008, el Comité Militar solicitó al Estado Mayor de la Unión Europea que liderara - con la asistencia de la Agencia Europea de Defensa y de otros actores relevantes en materia de gestión de crisis - la definición del Enfoque Integral e identificara sus posibles implicaciones militares ${ }^{58}$. Calificada como un área de capacidad

\footnotetext{
${ }^{53}$ Existen numerosos trabajos que centran su atención en analizar las provisiones contenidas en el Tratado de Lisboa en materia de política exterior y de seguridad; estudiar las atribuciones del Vicepresidente de la Comisión y Alto Representante de la Unión Europea para Asuntos Exteriores y Política de Seguridad o evaluar el potencial del Servicio Europeo de Acción Exterior. Ejemplos de ello pueden hallarse en: Missiroli, Antonio (2008): The Impact of the Lisbon Treaty on ESDP, XPO/B/SEDE/2007/50, Bruselas, Parlamento Europeo; Biscop, Sven y Algieri, Franco (2008): The Lisbon Treaty and ESDP: Transformation and Integration, Bruselas, The Egmont Institute; o Margaras, Vasilis (2010): Common Security and Defence Policy and the Lisbon Treaty Fudge: no Common Strategic Culture, no Major Progress, Working Paper $n^{\circ}$ 28, Bruselas, European Policy Institutes Network.

${ }^{54}$ Consilium: 3078th Foreign Affairs Council Meeting Conclusions on Civilian CSDP Capabilities, 8032/11, 21 marzo 2011, para 3.

${ }^{55}$ Parlamento Europeo: Resolution of 23 November 2010 on civil-military cooperation and the development of civilian-military capabilities, 2010/2071(INI), 23 noviembre 2010, para. 6. Esta idea puede también hallarse en otras fuentes como las que se citan a continuación: Gavas, Mikaela y Koeb, Eleonora (2010): Setting up the European External Action Service: Building a Comprehensive Approach to EU Action, Londres, Overseas Development Institute - European Centre for Development and Policy Management; Adebahr, Cornelius (2011): The Comprehensive Approach to Crisis Management in a Concerted Weimar Effort, Genshagen, Genshagen Foundation o Drent, op. cit., p. 11.

${ }^{56}$ Gebhard, op. cit., p. 13; Knutsen, op. cit., p. 5; Major y Mölling, op. cit., pp. 22-23; Norheim-Martinsen, op. cit., p. 7 o Hynek, op. cit., p. 3.

${ }^{57}$ Elaborada para implementar el análisis prospectivo Long Term Vision (2006) sobre las necesidades de la defensa europea, esta iniciativa conjunta entre la Agencia Europea de Defensa, el Comité Militar de la Unión Europea y los Estados miembros se fundamenta en las áreas de capacidad identificadas por el Headline Goal 2010 y la estimación de las que podrán requerirse en el 2025 para guiar la generación de nuevas capacidades susceptibles de ser empleadas en toda la gama de las operaciones PCSD. En el plan original aprobado en el año 2008, el Enfoque Integral y sus implicaciones militares constituía una de las doce grandes áreas de capacidad prioritarias para la Unión Europea. Sin embargo, en la revisión de 2011, el Comprehensive Approach es considerado como el motor principal y un área trasversal para el desarrollo de nuevas capacidades civiles y militares al servicio de la PCSD. European Defence Agency [EDA] (2012): Capability Development Plan (CDP) Fact Sheet, en: www.eda.europa.eu/Libraries/Documents/Capability_Development_Plan_fact_sheet.sflb.ashx.

${ }^{58}$ Carta del Estado Mayor de la Unión Europea a la Agencia Europea de Defensa, EUMS Support to the First Tranche of CDP results, 22 septiembre 2008
} 
prioritaria para la acción exterior europea ${ }^{59}$, el Comprehensive Approach se encaminaría a mejorar tanto la coordinación de los instrumentos internos de la Unión como las relaciones que ésta debería mantener con otras organizaciones internacionales - en especial las Naciones Unidas, la Alianza Atlántica, la Organización para la Seguridad y la Cooperación en Europa o la Unión Africana - en materia de prevención de conflictos, gestión de crisis, mantenimiento de la paz, estabilización postconflicto o construcción nacional ${ }^{60}$.

De esta forma, aunque desde una perspectiva política tiende a presentarse el Enfoque Integral como el marco general de actuación de la Unión Europea en materia de acción exterior y base para la gestión de crisis, en sentido estricto éste constituye el motor principal y un área trasversal para el desarrollo de nuevas capacidades civiles y militares al servicio de la PCSD $^{61}$. Además, atendiendo a la esperada Comunicación Conjunta de la Comisión Europea y el Alto Representante para la Política Exterior y la Seguridad Común sobre el Enfoque Integral $^{62}$ y las conclusiones del Consejo Europeo del pasado diciembre ${ }^{63}$ - que consideran urgente su desarrollo para incrementar la efectividad, visibilidad e impacto de la PCSD - es probable que Bruselas presente su propio concepto de Comprehensive Approach a lo largo del año 2014 siguiendo los pasos de la Alianza Atlántica, que en el 2010 emplazó esta iniciativa en la cúspide de su transformación militar, en uno de los fundamentos del nuevo Concepto Estratégico Compromiso Activo, Defensa Moderna y en el eje sobre el que se articulan las operaciones de gestión de crisis no-Artículo $5^{64}$.

No obstante, aunque las autoridades europeas han planteado en múltiples ocasiones la necesidad de adoptar un Enfoque Integral a la seguridad y han expuesto que sus actividades en materia de gestión de crisis se basan en la práctica en esta concepción - los ejemplos comprenden desde la lucha contra la piratería en el Cuerno de África y la estabilización de

\footnotetext{
59 Para facilitar los procesos de Desarrollo de Conceptos y Experimentación (Concept Development \& Experimentation), Investigación y Tecnología (Research \& Technology) y colaboración OTAN-UE en materia de capacidades militares, Bruselas ha asumido la definición aliada según la cual una capacidad - tanto militar como civil - combina Doctrina, Organización, Adiestramiento, Material, Liderazgo y Educación, Personal, Instalaciones e Interoperabilidad (NSA (2010): AAP-6 Glossary of Terms and Definitions, Bruselas, OTAN).

${ }^{60}$ Nicoletta Pirozzi (2013): The EU's Comprehensive Approach to crisis Management, Bruselas, Geneva Centre for Democratic Control of the Armed Forces; Drent, op. cit., pp. 11-13; Adebahr, op. cit., pp. 5-6 o Gavas y Koeb, op. cit., pp. 9-12.

${ }^{61}$ Así lo entiende el Plan para el Desarrollo de Capacidades de 2011 (EDA, op. cit., p. 1).

${ }^{62}$ Alto Representante de la Unión Europea para Asuntos Exteriores y Política de Seguridad: Joint Communication to the European Parliament and the Council - The EU's comprehensive approach to external conflict and crises, JOIN (2013) 30, 11 diciembre 2013, en: www.eeas.europa.eu/statements/ docs/2013/131211_03_en.pdf. Por otro lado, un análisis detallado del mismo puede hallarse en: Giulia Tercovich y Joachim Koop (2013): Assessing the EU's Joint Communication on the Comprehensive Approach: implications for EU crisis response and conflicto prevention, Bruselas, Global Governance Institute.

${ }^{63}$ Consejo Europeo: European Council 19/20 December 2013, 20 diciembre 2013, en: www.consilium. europa.eu/uedocs/cms_data/docs/pressdata/en/ec/140214.pdf.

${ }^{64}$ Jakobsen, Pete: “NATO's Comprehensive Approach after Lisbon: Princial Problem Acknowledged, Solution Elusive”, en Ringsmose, Jens y Rynning, Sten (eds.) (2011): NATO's New Strategic Concept: a Comprehensive Assessment, Copenhagen, Danish Institute for International Studies, pp. 83-90. Exacto, el Concepto Estratégico de 2010 fija tres funciones básicas para la Alianza Atlántica: la defensa colectiva, la seguridad cooperativa y la gestión de crisis. Para esta última se argumenta que la OTAN primará la prevención de conflictos (aunque la experiencia muestra que las diferencias entre los socios lo hacen improbable, tanto en el marco aliado como mediante cualquier posible colaboración entre ésta y la Unión Europea) y que intervendrá durante todo el ciclo de la gestión de crisis - prevención, resolución, estabilización posconflicto y apoyo a construcción - mediante un Enfoque Integral basado en la armonización de los medios militares y civiles a disposición de la OTAN y su coordinación con otros actores - incluida la Unión Europea, aunque el Concepto Estratégico la considerada como un socio (partner) más puesto que el vínculo trasatlántico se corresponde con las relaciones bilaterales entre los países europeos y americanos y no entre ambas organizaciones - durante el análisis previo, planeamiento, ejecución y evaluación de la gestión de la crisis.
} 
Somalia, las labores de adiestramiento en Mali para pacificar el Sahel o las actividades diplomáticas para resolver la guerra civil siria ${ }^{65}$ - a fecha de hoy Bruselas todavía no ha facilitado ningún detalle específico sobre su configuración, alcance o características más allá de las líneas planteadas por la Comunicación Conjunta y avaladas por el Consejo Europeo de diciembre de 2013. Así, en términos generales se asume que el Enfoque Integral debe armonizar todas las dimensiones de la acción exterior europea y aplicarse en todas las fases del conflicto (desde la alerta temprana hasta la gestión de crisis y la construcción de la paz) e implicar tanto a los Estados miembros como a las instituciones europeas ${ }^{66}$. Y para lograr este conjunto de objetivos - que deberían alcanzarse mediante la simplificación de la actividad interna de la Unión Europea en línea con los cambios orgánicos y funcionales establecidos por el Tratado de Lisboa y el reforzamiento de su dimensión exterior en todas las fases y planos de la operación ${ }^{67}$ - la Comunicación Conjunta fija la necesidad de trabajar en las siguientes dimensiones:

- Establecer mecanismos que permitan a los socios europeos obtener un análisis compartido de la crisis emergente y facilitar la identificación de los intereses, objetivos, recursos y papel de la Unión Europea en su prevención o resolución.

- Lograr que todos los países comparten la misma visión estratégica del conflicto con el objeto de articular una respuesta coherente a la crisis.

- Priorizar la prevención de conflictos mediante el empleo de herramientas diplomáticas o de alerta temprana.

- Movilizar los medios y capacidades políticas, económicas, diplomáticas, civiles y militares europeas para apoyar los objetivos compartidos.

- Proyectar cualquier actividad de prevención o respuesta a crisis a largo plazo y asegurar las sinergias entre los distintos instrumentos a disposición de la Unión Europea para garantizar una paz duradera.

- Mejorar la coordinación entre la dimensión interna y la vertiente exterior de la Unión Europea en materia de seguridad energética, protección medioambiental, contraterrorismo, crimen organizado y gobernanza económica.

- Emplear las delegaciones europeas para coordinar el diálogo y el apoyo europeo en el teatro de operaciones.

\footnotetext{
${ }^{65}$ Para los asuntos teóricos, véase Driver, op. cit., 139-141 o Van Osch, Tom (2011): A Comprehensive CivilMilitary Approach to Crisis Management, the role of the EUMS, Dublin, Institute of International European Affairs; mientras que para los asuntos del Sahel y el Cuerno de África puede observarse Pirozzi, op. cit., pp. 1317; Catherine Ashton: "Stop the Pirates, Help the Horn of Africa", European Voice, 23 febrero 2012, p. 9 o Hagström, Eva; Tham, Magdalena y Skeppström, Emma (2012): The EU Comprehensive Approach towards Somalia, Estocolmo, FOI. Finalmente, para la crisis siria, véase High Representative of the European Union for Foreign Affairs and Security Policy: Joint Communication - Towards a Comprehensive EU Approach to the Syrian Crisis, JOIN(2013)22, 24 junio 2013.

${ }^{66}$ No obstante, además de esta Comunicación Conjunta, es interesante observar también la declaración del Alto Representante Catherine Ashton sobre la revisión del Servicio Europeo de Acción Exterior, A314/13, 12 junio 2013.

${ }^{67}$ Drent, op. cit., p. 11 o Gavas y Koeb, op. cit., pp. 9-13; Wittkowsky, Andreas y Pietz, Tobias (2013): Under construction - The EU's Comprehensive Approach, Berlín, Center for International Peace Operations; y para observar algunas líneas generales, véase: Consilium: Promoting Synergies between the EU Civil and Military Capability Development, 15475/09, 9 noviembre 2009.
} 
- Incrementar la colaboración práctica entre la Unión Europea y el resto de actores internacionales y la sociedad civil.

A pesar de estas líneas generales que permiten encuadrar el Enfoque Integral europeo pero no aportan ningún detalle específico sobre su configuración práctica, es interesante comentar que hasta día de hoy han trascendido tres borradores del concepto, todos ellos centrados en las vertientes militar y operativa del Comprehensive Approach. Así, mientras el primero presenta las pautas, estructuras y procedimientos que deberían orientar su aplicación práctica en el teatro de operaciones ${ }^{68}$, el segundo busca conocer las implicaciones que su adopción puede tener sobre el análisis previo, el planeamiento, la conducción, el sostenimiento y la evaluación de las operaciones integradas civiles-militares ${ }^{69}$. Finalmente, el tercero - y probablemente el más controvertido a tenor de las revisiones realizadas sobre el trabajo base - determina las implicaciones operativas del Enfoque Integral y detalla tanto los protocolos de coordinación como las medidas de colaboración que deberían establecerse entre la Unión Europea y el resto de actores relevantes - en especial con las Naciones Unidas, la Organización para la Seguridad y Cooperación en Europa, la Alianza Atlántica o la Unión Africana - en el análisis previo, planeamiento y conducción de operaciones de gestión de crisis ${ }^{70}$.

Este último elemento reviste de una enorme importancia, especialmente tras la consolidación del Enfoque Integral en la Alianza Atlántica ${ }^{71}$ y su determinación por desarrollar “...una capacidad civil apropiada pero modesta"72 susceptible de facilitar la ejecución de cualquier operación integrada y reforzar la colaboración entre esta organización y los actores civiles en la zona de operaciones. En consecuencia, Bruselas deberá analizar con sumo detalle su proyecto de Enfoque Integral - especialmente después de que el Reino Unido alertara sobre la inconveniencia de crear nuevas estructuras y la necesidad de mantener la división de tareas con la Alianza Atlántica, donde ésta formalmente se encargaría de la vertiente militar de la gestión de crisis y la Unión de los aspectos civiles ${ }^{73}$ - y presentar un modelo que refuerce la coordinación interna de la Unión Europea y optimice su unidad de acción en materia de acción exterior mientras se armoniza, en la medida de lo posible, con el Comprehensive Approach aliado y su voluntad por dotarse - contra todo sentido común dada su naturaleza político-militar - de una limitada capacidad civil $^{74}$.

\footnotetext{
${ }^{68}$ Estado Mayor de la UE: A Military Perspective on a Comprehensive Approach for EU-led operations at theatre level, 1278/09, 12 febrero 2009.

${ }^{69}$ Estado Mayor de la UE: EUMS Food for thought paper - Operating Implications for a Comprehensive Approach for EU-led operations at theatre level, 1883/1/09, 19 mayo 2009.

${ }^{70}$ Estado Mayor de la UE: Towards a Comprehensive Approach. The Operating Implications, 9830/10, 17 mayo 2010; Estado Mayor de la UE: Towards a CA - the Operating Implications, 13093/09REV2, 9 marzo 2010 y Estado Mayor de la UE: Towards a CA - the Operating Implications, 13093/09, 13 diciembre 2009.

${ }^{71}$ Recuérdese que si bien el Comprehensive Approach aliado comenzó a desarrollarse tras la Cumbre de Riga (2006) y ha sido formalmente empleado en Afganistán; sus fundamentos conceptuales se hallan en el Enfoque a las Operaciones Basado en Efectos (Effects Based Approach to Operations) iniciado tras la Cumbre de Estambul (2004) y en el Planeamiento y Acción Concertados (Concerted Planning \& Action) de 2006. Un análisis más detallado de esta iniciativa puede hallarse en: Colom, op. cit., pp. 293-298.

${ }_{72}$ Declaración final de la Cumbre de Lisboa (20 de noviembre de 2010), para. 9.

${ }^{73}$ Carta del Reino Unido al Alto Representente de la Unión Europea en Asuntos Exteriores y Política de Seguridad, Putting the Comprehensive Approach to work, 18 noviembre 2011.

74 Algunos de los ejes que podrían articular estas nuevas relaciones entre la Alianza Atlántica y la Unión Europea pueden hallarse en: Hamilton, Daniel y Burwell, Francis (eds.) (2010): Shoulder to Shoulder: Forging a Strategic US-EU Partnership, Washington, Center for Transatlantic Relations.
} 
No obstante, es previsible que cualquier coordinación y/o división de tareas entre ambos actores sea más formal que real ya que primero debería superarse la dependencia militar europea de la Alianza Atlántica y de Estados Unidos, la predilección europea por emplear la OTAN en labores de gestión de crisis para forzar la participación militar de Washington, la variedad de enfoques existentes en el seno de ambas organizaciones, las manifiestas carencias europeas en materia civil que refutan su pretendida excelencia en este ámbito, la perversión de una división de tareas basada en una Alianza Atlántica - y más específicamente algunos de sus miembros - realizando acciones de alto riesgo y una Unión Europea - junto con algunos socios europeos de la Alianza - participando solamente en labores de apoyo a la paz, o las espectaculares desigualdades en materia de medios, capacidades o voluntad entre ambas organizaciones. Todos estos factores limitan las posibilidades de estructurar un Enfoque Integral europeo realmente útil y efectivo en materia de prevención de conflictos y gestión de crisis.

Llegados a este punto, es importante destacar que si bien para la elaboración de estos trabajos el Estado Mayor de la Unión Europea ha contado, desde el primer momento, con una amplia participación civil - caso del Directorado General para la Política Europea de Seguridad y Defensa (DG E VII), el Directorado General para Asuntos de Defensa (DG E VIII), el Directorado General para la Gestión de Crisis Civiles (DG E IX) o la Célula de Planeamiento y Conducción Civil - ninguna agencia civil europea se ha manifestado sobre los aspectos no-militares del Enfoque Integral. Esta realidad es un tanto paradójica, puesto que $a$ priori las principales ventajas cualitativas de la Unión Europea frente a la Alianza Atlántica no sólo son su autoproclamada vocación civil y normativa o su predilección por actuar junto con otros actores internacionales en el marco de un "multilateralismo eficaz", sino también la tenencia de una amplia gama de capacidades civiles (diplomáticas, económicas, de ayuda al desarrollo, policiales, judiciales, informativas, políticas...) que son fundamentales para la satisfactoria implementación de cualquier Enfoque Integral en la prevención de conflictos, la resolución de crisis, la estabilización posbélica o la construcción nacional.

\section{Conclusiones}

Considerado como el nuevo enfoque a la seguridad multidimensional y el paradigma para resolver cualquier crisis compleja, el Comprehensive Approach pretende concertar las estrategias, los recursos y las actividades de los distintos participantes en la gestión del conflicto a todos los niveles, fases y planos de la operación, desde su concepción inicial y posterior planeamiento operativo hasta su ejecución sobre el terreno y final evaluación.

La Unión Europea - gracias a su concepción integral y extensiva de la seguridad, vocación de potencia civil, normativa y multilateralista, predilección por el poder blando y variedad de instrumentos a su disposición - está especialmente capacitada para utilizar este modelo en su vertiente exterior y de seguridad. Es por esta razón que desde el año 2008 se halla articulando su propia concepción de Enfoque Integral para resolver sus carencias en materia de seguridad, ampliar la más restringida Coordinación Civil-Militar; explotar la nueva arquitectura institucional perfilada por el Tratado de Lisboa, unificar la dimensión civil y militar de la gestión de crisis o mejorar la efectividad, visibilidad e impacto de su acción exterior.

Y es que tal y como ha expuesto este trabajo, el Enfoque Integral constituye la tercera y más ambiciosa iniciativa - tras una Cooperación Civil-Militar encaminada a mejorar la 
colaboración ad-hoc a escala táctica y una Coordinación Civil-Militar enfocada a mejorar la coherencia interna de la Unión Europea en toda la gama de misiones Petersberg - propuesta por Bruselas para mejorar su unidad de acción en materia de política exterior y prevención de conflictos, ampliar su cooperación práctica con el resto de los actores implicados en la resolución de crisis y apoyar el desarrollo de capacidades civiles y militares al servicio de la PCSD.

Sin embargo, aunque tal y como ha ratificado el Consejo Europeo de diciembre de 2013 el Enfoque Integral constituye una de las principales iniciativas de la Unión Europea en materia de acción exterior y de seguridad para incrementar la efectividad, visibilidad e impacto de la PCSD, todavía no parece existir ningún denominador común sobre cómo debe configurarse específicamente este modelo más allá de la obtención de una visión común, de definición de una estrategia integral, la colaboración entre los socios comunitarios o la armonización de los instrumentos al servicio de Bruselas para ofrecer una acción coherente en la acción exterior, prevención de conflictos, gestión de crisis o fortalecimiento de la paz.

Y cuando finalmente se presente un concepto que expanda y detalle las líneas de acción fijadas en la reciente Comunicación Conjunta sobre el Enfoque Integral, éste no sólo deberá salvar los mismos escollos que la Coordinación Civil-Militar - armonizar los procesos de toma de decisiones, regularizar las prioridades operativas de los elementos civiles y militares, garantizar la colaboración de los activos europeos en todos los niveles y fases de la misión, mediar en las eternas luchas internas o integrar las distintas culturas organizativas que existen en Bruselas - sino también conciliar los intereses de los Estados miembros; garantizar una difícil unidad de acción en materia de política exterior, coordinar las actividades de gestión de crisis con otras organizaciones internacionales y salvaguardar el statu quo - bien comporte la complementariedad, la duplicación racional o la división de tareas ${ }^{75}$ - con la Alianza Atlántica, su Enfoque Integral y su determinación por dotarse de una dimensión civil fuera de todo sentido común.

Aunque estos obstáculos - debidos fundamentalmente a la idiosincrasia europea, las prioridades de los Estados miembros y las complicadas relaciones entre la Alianza Atlántica y la Unión Europea - posiblemente dilatarán la implementación práctica del Enfoque Integral; las disposiciones contenidas en el Tratado de Lisboa, el lanzamiento del Servicio Europeo de Acción Exterior y la entrada en funcionamiento del Directorio para el Planeamiento y Gestión de Crisis, coadyuvarán para que el Enfoque Integral europeo se convierta pronto en una realidad formal o efectiva.

Solamente el futuro demostrará si el Enfoque Integral europeo puede solventar estas carencias en materia de prevención de conflictos y gestión de crisis. No obstante, situaciones como las de Libia, con una coalición ad-hoc franco-británica, la oposición frontal de varios socios comunitarios y sin ningún planteamiento para estabilizar el conflicto; Siria con una falta de visión común sobre el problema o las medidas a tomar y con París determinado a participar en un ataque junto a Washington; o Mali donde a pesar de reconocer la importancia de la región y lanzar una misión de adiestramiento, el peso de intervención lo llevó Francia, parecen comprometer la utilidad práctica de esta iniciativa que pretende armonizar las labores de prevención de conflictos y gestión de crisis en el seno de la Unión Europea. Y aunque los ejemplos de Libia, Siria o Mali fueran casos aislados, el Enfoque Integral europeo deberá

\footnotetext{
75 Muñoz Enrique y Ortiz, Enrique (2010): El Nuevo Concepto Estratégico de la OTAN: una aportación española, Documento de Trabajo no 49/2010, Madrid, Fundación Alternativas, Madrid, pp. 50-54 o Colom, Guillem: "La Alianza Atlántica ante el nuevo Concepto Estratégico", Revista de Estudios Europeos, no 59 (julio 2012), pp. 41-60.
} 
aplicarse en un momento en el que Europa parece cada vez más ajena a los asuntos estratégicos globales, Estados Unidos se está despreocupando del continente, ha adoptado un enfoque muy selectivo a su acción exterior y ha repetido en innumerables ocasiones que ha llegado el momento de que Europa se responsabilice de su propia seguridad, los intereses nacionales de sus miembros parecen cada vez más incompatibles, las políticas de defensa europeas se están renacionalizando a pesar de las provisiones de la PCSD y, en muchas ocasiones, la voluntad de Bruselas por lograr una sola voz en la escena internacional parece más formal que real.

A pesar de que estos factores pueden ensombrecer el presente y el futuro del Enfoque Integral, con toda probabilidad esta iniciativa constituye la más importante y ambiciosa propuesta realizada por Bruselas para promover una cultura de coordinación civil-militar, integrar su acción exterior y adaptar la Unión Europea al complejo, heterogéneo y conflictivo mundo del siglo XXI. 